Revue d'histoire de l'Amérique française

DEVUE D.HISTOIRE DE L'AMÉRIQUE FRANÇAISE

\title{
La propagande de l'École sociale populaire en faveur du syndicalisme catholique 1911-1949
}

\section{Jean-Claude St-Amant}

Volume 32, numéro 2, septembre 1978

URI : https://id.erudit.org/iderudit/303690ar

DOI : https://doi.org/10.7202/303690ar

Aller au sommaire du numéro

Éditeur(s)

Institut d'histoire de l'Amérique française

ISSN

0035-2357 (imprimé)

1492-1383 (numérique)

Découvrir la revue

Citer cet article

St-Amant, J.-C. (1978). La propagande de l'École sociale populaire en faveur du syndicalisme catholique 1911-1949. Revue d'histoire de l'Amérique française, 32(2), 203-228. https://doi.org/10.7202/303690ar d'utilisation que vous pouvez consulter en ligne. 


\section{LA PROPAGANDE DE \\ L'ÉCOLE SOCIALE POPULAIRE EN FAVEUR DU SYNDICALISME CATHOLIQUE 1911-1949}

Jean-Claude St-Amant

La première publication de l'École Sociale Populaire paraît au mois de juillet $1911^{1}$. Cette "société de propagandistes sociaux", fondée par les Jésuites de Montréal, précise qu'elle a pour but «de travailler au salut du peuple et à l'amélioration de son sort, en propageant l'idée d'association catholique, surtout sur le terrain professionnel». Préoccupations spirituelles et temporelles qui, orientées surtout vers les travailleurs, se traduisent par le souci de les organiser. D'ailleurs, le deuxième article de son programme stipule «que l'organisation professionnelle, à personnalité civile et à base confessionnelle, est le meilleur moyen de conserver et de rétablir la paix sociale et d'améliorer le sort des travailleurs dans les villes». Jusqu'à la fin des années 40, l'École restera fidèle à cette vocation. Elle considère alors que les syndicats catholiques (la Confédération des Travailleurs catholiques du Canada) sont organisés sur des bases solides: ils publient leur journal et possèdent leur propre service de renseignements, d'étude et de formation des membres. L'École n'a donc plus de raison de continuer son œuvre dans ce domaine particulier. Elle en est consciente quand elle écrit en octobre 1947 que "le monde ouvrier a atteint sa majorité. Les travailleurs organisés sont devenus capables de réaliser par eux-mêmes leurs légitimes destinées. ${ }^{2} »$

1 Les renseignements et les citations donnés dans ce paragraphe sont tirés de la brochure: École Sociale Populaire, L'École Sociale Populaire: But, organisation, programme (Montréal, E.S.P. [1911], 20 p., coll. «E.S.P.», $\mathrm{n}^{\circ} 1$.

2 Jacques Cousineau, "Promotion ouvrière», Relations, 7, no 10 (octobre 1947): 294.

[203]

RHAF, vol. $32, n^{\circ} 2$, septembre 1978 
Fernand Dumont affirme que. «les syndicats catholiques [...] ont toujours emprunté aux idéologies dominantes ${ }^{3}$. Celles-ci émanent en partie de la doctrine sociale de l'Église catholique. Dès 1914, Mgr Paul Bruchési avait confirmé l'École Sociale Populaire (E.S.P.) dans sa vocation de centre d'études et de propagande du syndicalisme catholique. Ainsi, ses écrits représentent la doctrine sociale de l'Église en matière syndicale, telle que conçue et adaptée pour la société canadienne-française.

Notre propos dans les quelques pages qui vont suivre sera d'une part de montrer l'ampleur du réseau de propagande jésuite et d'autre part de définir la doctrine syndicale qu'elle a diffusée.

\section{I - L'École Sociale Populaire: origine et évolution}

À Montréal, l'action concrète de l'Église en milieu ouvrier tarde un peu. Le caractère cosmopolite de la ville semble poser des problèmes particuliers et il faut attendre 1909 avant de voir un début d'organisation de l'apostolat ouvrier. Deux congrès successifs de la Fédération des Ligues du Sacré-Cœur, un organisme apostolique nouvellement formé, aboutissent aux résolutions suivantes:

1. Il est nécessaire et possible d'unir nos ouvriers dans des syndicats professionnels nettement catholiques.

2. Là où la place est libre, et le terrain bien préparé, on peut dès maintenant fonder ces syndicats.

3. Il faut avant tout faire l'éducation sociale du clergé et des autres classes de la société.

4. Pour étudier ce programme et continuer le travail commencé, le Congrès se forme en comité permanent et charge son $\mathrm{Bu}$ reau de Direction de préparer un projet d'organisation ${ }^{4}$.

Du projet d'organisation émerge l'École Sociale Populaire qui se définit comme centre de recherche et de documentation. Les statuts officiels sont approuvés par l'archevêque de Montréal, Mgr Paul Bruchési, en avril 1911.

3 Fernand Dumont, "Du début du siècle à la crise de 1929: un espace idéologique», dans Fernand Dumont et al, Idéologies au Canada français (Québec, Les Presses de l'Université Laval, 1974), 7.

4 Cité dans M. Têtu, Les premiers syndicats catholiques canadiens (19001921), Thèse de doctorat, Université Laval, 1961, 27. 


\section{La première organisation}

L'École est constituée d'un président et d'un vice-président d'honneur (deux religieux dont l'archevêque), d'un bureau de direction composé de douze membres (sept religieux et cinq laïcs) et de quatre comités permanents (trois religieux et six laïcs, en tout) s'occupant des études et des publications, de la propagande, des finances et d'action ouvrière. Une souscription de 500 dollars est nécessaire pour devenir «membre d'honneur fondateur» tandis que 100 dollars assurent le titre de «membre d'honneur bienfaiteur». Ces coûts très élevés limitent, il est certain, le nombre de membres d'honneur. Une contribution annuelle d'un dollar suffit pour devenir «membre actif» et recevoir les publications de l'École.

En réalité, le personnel est beaucoup plus réduit que ne le laisse supposer cette organisation ${ }^{5}$. Seulement deux personnes y travaillent activement: le jésuite Léonidas Hudon et son secrétaire Arthur Saint-Pierre. Ceux-ci réussissent tout de même à former quelques syndicats dans Montréal, à monter une bibliothèque accessible au public, à organiser quelques cours et conférences dans différents centres de la province, et à publier une brochure mensuelle.

En marge de cette activité propre à l'E.S.P. - et identifiée comme telle - les Jésuites établissent ou collaborent à plusieurs autres mouvements, ligues et associations. Ils constituent un véritable réseau communautaire. Pendant les premières années, l'activité la plus importante reste la publication du Bulletin paroissial ${ }^{6}$ et du Messager du Sacré-Cœur. En 1901, l'imprimerie du Messager avait été déménagée au scolasticat de l'Immaculée-Conception, maison qui héberge également une cinquantaine de Pères jésuites dont environ la moitié se voue à «l'apostolat de la plume»: ils rédigent de nombreux textes qui servent à alimenter le réseau de distribution.

$s$ J.-P. Archambault note à cet effet: «combien de ces vingt-trois membres purent apporter à l'œuvre une autre collaboration que celle de leur patronage ", Esquisses sociales (Montréal, Librairie d'Action française Ltée, 1930), 125.

6 Jean Blouin, «Un véritable réseau d'éducation populaire et d'action sociale», Perspectives, 28 juin 1975. Selon l'auteur, le Bulletin a rejoint jusqu'à 140000 familles.

Joseph-Papin Archambault ajoute qu'«il existe [...] au Canada [en 1918], environ 225 bulletins paroissiaux de langue française. Deux cent douze d'entre eux ont une partie commune [...] rédigée par un Père jésuite de l'Immaculée-Conception, Le clergé et l'Action sociale (Montréal, E.S.P., 1918), 84. 
Le manque de ressources humaines ainsi que des problèmes nouveaux suscités par la guerre ont pour effet de mettre en veilleuse les activités de l'École. En 1915, les Jésuites prennent officiellement en main le centre et réduisent ses activités à la publication des brochures mensuelles et à quelques conférences.

L'arrivée de Joseph-Papin Archambault permet à l'organisme de prendre un nouvel essor. Les activités de celui-ci nous livrent l'orientation de l'École pour les années à venir.

\section{L'influence du père Joseph-Papin Archambault}

Le père Archambault n'est pas nouveau sur la scène du catholicisme social au Québec. Il a participé en 1909 à l'organisation des retraites fermées, une œuvre religieuse dont la portée se fera aussi sentir chez les syndicats catholiques; trois ans plus tard, il a fondé la ligue des Droits du français avec Joseph Gauvreau, Omer Héroux et quelques nationalistes disciples de Henri Bourrassa ${ }^{7}$. Après une année d'études spirituelles en Europe (1913), on le retrouve à l' $A c$ tion française (1917), principal organe nationaliste de l'époque, à la direction de La Vie Nouvelle (1918), maison d'édition où est publiée la revue du même nom ainsi qu'un almanach, et à la direction de L'Oeuvre des tracts (1919) où paraît une petite brochure mensuelle à caractère religieux. Il est enfin le principal promoteur et le directeur des Semaines sociales inaugurées à Montréal en 1920, sous les auspices de l'École Sociale Populaire.

L'année suivante, le père Archambault réforme complètement les organismes qu'il dirige. Il en réunit l'administration et la rédaction sous deux comités centralisés. L'Oeuvre des tracts, les brochures mensuelles de l'E.S.P., ou les Semaines sociales gardent cependant leur identité propre. L'économie d'argent et de personnel ainsi réalisée, de même que l'utilisation d'un périodique pour faire la publicité de l'autre, assurent à l'œuvre un succès qui ne se démentira pas pendant plusieurs années.

Les renseignements sont très épars sur les collaborateurs de l'École entre la fin de la première guerre mondiale et la fondation de L'Ordre nouveau en 1936. Lors du vingt-cinquième anniversaire de

7 Le Devoir accordera d'ailleurs son appui aux syndicats catholiques, notamment dans une série de quatorze articles de Bourassa publiés du 15 avril au 7 mai 1919. 
l'E.S.P., le père Archambault avoue qu'il «n'oserait dévoiler sans rougir, le nombre de ceux qui sont attachés à l'École Sociale Populaire ${ }^{8}$. Entendons que leur nombre n'a jamais dépassé trois ou quatre personnes et que le père Archambault a souvent fait cavalier seul. Cette situation explique le grand nombre d'auteurs différents qui ont signé une brochure ou un tract: l'École se fiait sur un collaborateur occasionnel ou reprenait des textes publiés ailleurs. En ce sens, l'École joue vraiment le rôle d'un centre de propagande et de diffusion. Les nombreux services qu'elle offre à partir de 1933 contribue aussi à lui donner ce caractère particulier.

\section{Les ramifications du centre}

Au nombre des initiatives de l'École, la plus importante et la plus puissante reste sans doute son service de presse hebdomadaire, mis sur pied en 1933:

Elle [l'E.S.P.] adresse chaque semaine à tous les journaux de langue française du Canada [...] un bulletin d'actualité sociale [...] Grâce au communiqué qu'elle reçoit de plusieurs centres catholiques européens, il lui est facile d'exposer les initiatives intéressantes accomplies ailleurs et de redresser les nouvelles trop tendancieuses - en ce qui concerne, par exemple, la propagande communiste - des agences internationales. Presque tous les journaux utilisent ce bulletin 9 .

Quant à l'utilisation que les journaux en font, le journal poursuit:

quelques-uns le reproduisent tel quel, en mentionnant son origine; la plupart - et le procédé nous semble préférable - s'en assimilent la matière et le répartissent dans leurs colonnes suivant les sujets traités ${ }^{10}$.

Ce contrôle de l'information internationale a certainement contribué à influencer le lecteur dans le sens souhaité par l'École, puisque le communiqué en question «est consacré en bonne partie au communisme» ${ }^{11}$.

8 E.S.P., Les vingt-cinq ans de l'École Sociale Populaire 1911-1936 (Montréal, E.S.P., 1936), 45, coll. «E.S.P.» nos 269-270.

9 «Service de presse», L'Ordre nouveau, 3, no 17 (5 juin 1939).

10 Ibid.

11 J.-P. Archambault, "Les trois phases de l'École Sociale Populaire», Les vingt-cinq ans de l'École Sociale Populaire 1911-1936, p. 45. Le service de presse est disparu avec la guerre de 1939. 
L'E.S.P. entend aussi "former des chefs catholiques, surtout dans les milieux ouvriers ${ }^{12}$. Tel est le but que se fixe l'École de formation sociale organisée en 1931. Sous la direction de JosephPapin Archambault, l'École réunit sous un même toit, pendant une semaine, divers groupes «qui se livrent à une étude intensive de la doctrine sociale catholique et s'exercent à la propager dans leurs milieux ${ }^{13}$. Cours, études en commun, débats publics et exercices spirituels alternent avec des périodes de lecture dans la bibliothèque mise à leur disposition. Les participants se transforment ensuite en conférenciers dans les cercles d'étude, les clubs sociaux, les fédérations ou encore à la radio. Certains d'entre eux restent en contact par des réunions bimensuelles.

Parmi les professeurs invités, J.-B. Desrosiers et Gustave Sauvé sont régulièrement du nombre. S'y joignent aussi les pères G.-H. Lévesque, Louis Chagnon et Léon Lebel.

L'École de formation sociale tient trois ou quatre sessions par année, durant l'été, tout d'abord à Vaudreuil puis à Boucherville à partir de 1941. En 1938, une telle session regroupe 18 participants, dont plusieurs dirigeants du syndicalisme catholique (Philippe Girard $\mathrm{y}$ assiste) et deux employeurs ${ }^{14}$. En 1941, "les syndiqués constituaient le groupe le plus nombreux [...] ils comptaient quelques-uns des principaux chefs du syndicalisme national catholique au $\mathrm{Ca}$ nada» ${ }^{15}$. Deux ans plus tard, le groupe de syndicalistes présents semble assez important pour justifier la création d'une section spécifiquement syndicale où on aborde l'étude de sujets tels que la convention collective (1944) ou le mouvement syndical au Canada (1945). Le succès de la formule incite les Jésuites à étendre leur activité et Saint-Hyacinthe accueille une École d'action ouvrière en 1945. René Bergeron, conférencier anticommuniste de l'E.S.P. formé à Vaudreuil, est invité à prendre la parole à plusieurs de ces sessions. Les syndicats catholiques les organisent finalement euxmêmes à partir de 1946, tout en continuant à se servir de l'équipe de conférenciers de l'E.S.P.

Toujours dans le but de diffuser son message le plus largement possible, l'École organise à partir de 1934 des journées anticommu-

12 «L'École de formation sociale», L'Ordre nouveau, 2, no 17 (5 juin 1938).

13 "Initiative fructueuse", Relations, 2, no 8 (août 1942): 206.

14 «École de formation sociale», L'Ordre nouveau, 2, no 17 (5 juin 1938).

15 «Formation sociale», Relations, 1, no 7 (juillet 1941): 171. 
nistes dans les différentes villes importantes de la province ${ }^{16}$. Des conférences suivent l'exposition de documents communistes et la vente de brochures hors-série, de tracts et d'affiches anticommunistes. La même formule sert, à partir de 1942, à des «journées de formation syndicale» ou se transforme en des "semaines syndicales» et des "semaines syndicales-patronales». Ce genre de campagne comprend aussi «des articles de journaux et de revues, des causeries à la radio, des sermons dans les églises, des publications de brochures et de tracts ${ }^{17}$.

\section{Les périodiques publiés par l'École Sociale Populaire}

Les brochures mensuelles ont été publiées sans interruption pendant toute la période d'activité de l'École. Cette abondante documentation traite de questions sociales très diverses, passant de thèmes précis comme l'alcoolisme, le cinéma ou le logement à des sujets aussi vastes que «la restauration de l'ordre social» ou «l'organisation démocratique de la vie sociale». Quelques-unes réunissent sous un même titre de courtes études faites par des auteurs différents. L'E.S.P. «entend faire de ces brochures mensuelles une publication de premier ordre qui puisse rivaliser par la sûreté de son enseignement doctrinal et l'exactitude des faits exposés avec les meilleures du genre publiées à l'étranger ${ }^{18}$. Elles servent également à renseigner sur les différentes activités sociales catholiques.

De présentation très sobre et de format invariable, les brochures sont tirées «à plusieurs milliers d'exemplaires [...] quelques-unes ont même eu un tirage de trente ou quarante mille» 19 .

Dans le but d'augmenter leur circulation, chacune énumère tous les titres déjà parus et encore disponibles. Fréquemment, la dernière page fait de la publicité pour un volume nouvellement paru ou vante les mérites de certains périodiques catholiques.

16 L'E.S.P. consacre deux brochures au compte rendu de ces journées. Voir E.S.P., Journées anticommunistes I (Montréal, E.S.P., 1935), 64, coll. «E.S.P.», nos 251-252, et Journées anticommunistes II (Montréal, E.S.P., 1935), 26, coll. «E.S.P.», no 253 .

17 «Semaines syndicales», L'Ordre nouveau, 1, no 24 (20 septembre 1937). Le numéro entier est consacré à cette activité.

18 "Avis», L'Organisation professionnelle (Montréal, E.S.P., 1921), 1, coll. «E.S.P.», no 96.

19 Ibid., 2. Ces chiffres semblent exagérés. L'estimation de 8 à 9 milliers d'exemplaires du père d'Auteuil Richard semble plus près de la réalité. 
Parmi les auteurs connus alors, il faut dégager le nom des jésuites J.-P. Archambault, P. Fontanel, Adélard Dugré ainsi que ceux de Alfred Charpentier, d'Arthur St-Pierre, des abbés Edmour Hébert et Édouard-Eugène Gouin. Il faut surtout noter le très grand nombre de collaborateurs; $79 \%$ d'entre eux n'ont écrit qu'à une ou deux reprises. La majorité $(63 \%)$ fait partie du clergé. Cette proportion reste à peu près constante pendant toute la période. Par contre, si on exclut les auteurs réguliers (qui ont écrit deux fois ou plus), le nombre de collaborateurs laïques est sensiblement égal à celui des religieux.

Nous retrouvons à peu près les mêmes auteurs religieux dans le cas des tracts publiés par l'École; la coopération de laïcs est cependant beaucoup plus réduite. Bien que publiés tous les mois de 1919 à 1949 , les tracts n'ont pas connu le même succès que les brochures. Leur tirage reste à environ mille exemplaires. Ils portent exclusivement sur des questions religieuses: biographies de saint, œuvres apostoliques, etc., et leur couverture présente une image pieuse correspondant au sujet traité.

Par les Semaines sociales, l'E.S.P. se sert d'une approche très différente pour diffuser son enseignement. "[L'œuvre] s'efforce, par un enseignement à la fois doctrinal et pratique, de former une élite qui fera revivre l'esprit chrétien dans les mœurs, les institutions et les lois ${ }^{20}$. Les sessions réunissent dans une rencontre annuelle de cinq jours (une fin de semaine après 1940), tenue vers la fin de l'été dans diverses villes de la province, plusieurs conférenciers qui traitent de sujets rattachés à un thème central. Il s'agit souvent de certains points d'une encyclique papale sur laquelle on désire discuter. Trois cours durant la journée précèdent une «manifestation ouvrière» tenue dans un parc ou une salle publique le soir; en 1942, la manifestation est remplacée par un film. La matière abordée lors d'une semaine est choisie à la fin de la rencontre précédente. Les membres d'une commission générale s'entendent aussi sur le lieu et la date de la session suivante. Une rencontre ultérieure confirme la décision, propose des conférenciers et soumet le tout pour approbation finale à l'évêque du diocèse qui doit recevoir les semainiers.

La commission générale est constituée de personnes au nom prestigieux, des laïcs dans près de la moitié des cas. Ils donnent peu de conférences (en moyenne deux membres donnent une conférence à chacune des sessions) et sont en général peu actifs dans l'organisa-

20 «Les Semaines sociales», L'Ordre nouveau, 4, no 23 (5 septembre 1940). 
tion des semaines ${ }^{21}$; cette tâche revient principalement au père $\mathrm{Ar}$ chambault. Un commentaire de Lionel Groulx, bien qu'appliqué au travail du père Archambault à l'Action française, nous explique le rôle qu'il a tenu aux Semaines sociales: «Il [J.-P. Archambault] venait nous embrigader, nous arracher une conférence, un article, un tout petit article qui ne coûterait qu'un instant, un tour de plume, etc...» ${ }^{22}$. Le conférencier pressenti reçoit un «dossier» sur le sujet à développer ainsi qu'un plan schématique de la conférence. Le texte rédigé est ensuite soumis au père Archambault pour une dernière vérification avant d'être livré au public. Cette pratique lui permet de garantir l'orthodoxie des conférenciers: "C'est la vérité qui parle par leurs bouches ${ }^{23}$, assure-t-il. Encore dans ce cas-ci, la très grande diversité des conférenciers, autant clercs que laïcs, est remarquable. Sauf dans de très rares exceptions, ils ont exercé leur talent seulement à une ou deux reprises.

L'assistance aux Semaines varie beaucoup d'une année à l'autre, mais les quelques renseignements que nous possédons indiquent une nette croissance avec les années. L'auditoire a été estimé à deux ou trois cents personnes pendant une journée de 1920 et à environ 2000 lors d'une assemblée populaire en 1937. Ce sont «des étudiants [...], une nombreuse représentation de l'épiscopat de [la] province, des juges, des avocats, des hommes de toutes les classes de la société» ${ }^{24}$, selon un conférencier de 1947. À cet auditoire s'ajoute à quelques reprises le public de la radio durant les années 30 et suivantes; les comptes rendus in extenso que vend l'E.S.P. et les nombreux articles que font régulièrement plusieurs journaux québécois augmentent le rayonnement des Semaines sociales.

L'École tient tout de même à lancer son propre organe bimensuel en octobre 1936. "L'exposition de la doctrine sociale catholique constituera [son] principal effort ${ }^{25}$.» L'Ordre nouveau spécifie aussitôt que le communisme le préoccupe particulièrement:

21 Pour une liste des membres, voir Marie-Agnès de Rome Gaudreau, The Social Thought of French Canada as Reflected in the Semaine Sociale (Washington, The Catholic University of America Press, 1946), 31-62.

22 Lionel Groulx, «L'animateur de l'Action française », Lettres du Bas-Canada, 21, no 1 (mars 1967): 42 .

23 J.-P. Archambault, «L'Église et le syndicalisme», Semaines sociales du Canada (Montréal, L'Action paroissiale, 1921), 3. Il est important de remarquer que le texte dont nous disposons a aussi pu être «remanié » avant d'être publié.

24 Abel Marion, «L'organisation professionnelle protège la vie rurale», Semaines sociales du Canada (Montréal, E.S.P., 1947), 255. octobre 1936).

25 "A nos lecteurs. Notre but - Notre plan», L'Ordre nouveau, 1, no 1 (5 
Nous devons lui [le communisme] consacrer une attention particulière, et faire connaître non seulement son activité au Canada mais aussi sa doctrine, sa tactique, ses progrès dans le monde ${ }^{26}$.

Le Programme de restauration sociale lancé par l'École tiendra la deuxième place en importance dans ses pages.

Le tirage très bas du journal ( 2 à 3000 exemplaires) n'indique pas nécessairement l'influence qu'il a pu exercer. L'Ordre nouveau «ne recherche pas le grand nombre [...] c'est sur une élite qu'[il] veut agir, sur ceux qui tiennent les leviers de commande, sur les autorités sociales» ${ }^{27}$. La publication régulière de plans d'étude, de bibliographies et de catéchismes sous forme de questions et réponses indiquerait qu'il a été conçu pour le clergé québécois, les dirigeants d'œuvres sociales et les membres des cercles d'étude.

Les rédacteurs à L'Ordre nouveau font tous partie du clergé. Les pères Jacques Cousineau, Omer Genest, Joseph Ledit et J.-P. Archambault écrivent régulièrement. Ils sont aidés en septembre 1939 par l'arrivée de Jean d'Auteuil Richard qui a terminé deux ans d' «études sociales» en Europe. Il deviendra le membre le plus prolifique du groupe. Les abbés Philippe Perrier, Émile Cloutier et Jean Bertrand de même que Gérard Picard et Alfred Charpentier signent aussi quelques écrits de façon occasionnelle ou tiennent une rubrique de courte durée.

La publication de L'Ordre nouveau ne durera que quatre ans; le journal annonce, le 20 décembre 1940, la parution de la revue mensuelle Relations pour le mois suivant: «les esprits évoluent rapidement. Ceux d'aujourd'hui ont d'autres exigences que ceux d'hier [...] On veut des aliments substantiels et appropriés aux besoins actuels mais sous une forme légère, agréable, variée ${ }^{28}$. Le contenu s'ouvrira à des thèmes autrefois relégués au second plan:

[La question sociale] restera au premier plan. Mais elle n'excluera pas les autres questions: questions familiales, morales, nationales, questions d'éducation et d'action catholique, qui se posent de façon impérieuse dans notre pays. Ces questions sont intimement liées à l'ordre, à la justice, à la religion. Notre revue ne peut les ignorer ${ }^{29}$.

26 Ibid. La page trois est régulièrement consacrée au communisme.

27 Ibid.

28 L'Ordre nouveau, 5 , nos 5 et 6 (5 et 20 décembre 1940).

29 Ibid. La revue s'occupera surtout de la famille (éducation des enfants, travail des femmes) et de coopération. 
Elle ne s'adresse "ni à la masse ni à l'élite mais à tous ceux qui croient en la véritable démocratie» ${ }^{30}$. Par rapport à $L^{\prime} O r d r e$ nouveau, Relations tente de diversifier sa clientèle. Elle entend «analyser les courants et les contre-courants de l'opinion mondiale en autant qu'ils intéressent le Canada et le Canada français, poser les problèmes que comportent les relations des hommes et les résoudre en s'inspirant de la doctrine authentique de l'Église et de l'esprit chrétien ${ }^{31}$.

Ces projets laissent cependant transparaître un certain sentiment d'inquiétude devant l'évolution de la société, sentiment qui suivra Relations même après la fin du second conflit mondial: "la menace pèse sur l'homme, plus largement et plus intimement: les valeurs de notre civilisation et de notre culture sont en jeu, la crise est dans les relations de l'homme à l'homme» ${ }^{32}$. L'ampleur et la difficulté des problèmes rendent les rédacteurs inquiets.

L'équipe de rédaction est composée au début de Joseph-Papin Archambault (directeur jusqu'en juin 1941), de Jean d'Auteuil Richard (directeur de juillet 1941 à décembre 1948), de Jacques Cousineau, Joseph Ledit, Alexandre Dugré, Émile Bouvier, Luigi d'Appollonia et Omer Genest. Ces Jésuites sont tous des collaborateurs déjà rencontrés précédemment à l'E.S.P.

Le tirage de la revue indique un net progrès, passant de 1000 abonnements payés en 1941 à 10000 en 1946 et 14500 en 1949.

\section{Les sources d'inspiration du centre}

À ses débuts, l'E.S.P. se réclame, dans son action, de l'Action Populaire de Reims. Ce mouvement social catholique avait été créé en France par les Jésuites à la fin du siècle dernier. Les buts visés et les moyens utilisés se retrouvent quasi intégralement à l'E.S.P. Ainsi en est-il de la publication d'une brochure mensuelle; la pratique a simplement été transplantée au Québec.

L'influence n'est pas que de forme. Elle touche aussi le contenu. Plusieurs auteurs citent abondamment les brochures françaises; quelques-unes d'entre elles sont reproduites intégralement.

\footnotetext{
30 «Présentation», Relations, 1, no 1 (janvier 1941): 2.

31 Ibid: 1.

32 Ibid.
} 
L'échange de publications devient constant, comme en témoigne cette publicité de L'Ordre nouveau:

L'École Sociale Populaire a fait venir de France et de Belgique plusieurs tracts que nous demandons à nos amis de répandre dans leurs milieux, ceux surtout qui sont travaillés par la propagande communiste ${ }^{33}$.

L'École pousse plus loin encore sa collaboration. Elle avoue avoir

puisé à l'Action Populaire son inspiration et plusieurs de ses initiatives. Elle a reçu une aide constante, soit sous forme de professeurs ..., soit sous forme de conseils, de publications, d'hospitalité même [...] Actuellement, depuis près de deux ans, un Jésuite canadien-français qui se destine à l'E.S.P. est l'hôte fortuné de l'Action Populaire ${ }^{34}$.

Les Semaines sociales ont aussi eu leurs prédécesseurs européens. Elles existent en Allemagne (depuis 1893), en France, en Espagne, en Belgique et même dans quelques pays d'Amérique du Sud. De l'aveu du père Archambault, celles du Canada sont calquées sur le modèle français.

L'influence française se fait aussi régulièrement sentir au niveau du contenu des Semaines. Il suffit pour s'en rendre compte de comparer les sujets abordés lors des Semaines de 1921 au Canada et de 1907 en France, celles de 1925 et 1921, de 1927 et 1925, de 1936 et 1935 , de 1945 et 1938 pour les mêmes pays respectivement. De même, plusieurs discours prononcés au Québec donnent crédit à leurs sources françaises, les citent régulièrement ou s'en inspirent ouvertement. Enfin, certains conférenciers de l'École viennent directement d'outre-mer. C'est le cas du chanoine Des Granges en 1914 qui s'emportera contre le socialisme et les syndicats internationaux, et du chanoine Rutten l'année suivante.

Bref, de 1911 à 1949, les témoignages d'une collaboration étroite entre les deux institutions abondent. Seuls les textes de la revue Relations semblent garder une indépendance vis-à-vis des publications françaises. Il n'en demeure pas moins que quelques-uns de ses rédacteurs ont été formés en France et que, finalement, ils puisent aux mêmes sources doctrinales:

33 L'Ordre nouveau, 1 , nos 19 et 20 (5 et 20 juillet 1937). Ce genre de publicité est très fréquent.

${ }_{34}$ L'Ordre nouveau, 3, no 9 (5 février 1939). Le Jésuite mentionné est Jean d'Auteuil Richard. 
C'est à la lumière des enseignements de Rome que nous entendons nous éclairer, ce sont les leçons contenues dans les encycliques [...] que nous voulons nous efforcer de faire pénétrer davantage dans les esprits et dans les lois ${ }^{35}$.

Cette constatation du père Archambault, faite lors de la session de 1936 des Semaines sociales, conviendrait tout autant en 1911 qu'en 1949. À preuve, la première Semaine sociale est consacrée entièrement à l'encyclique Rerum Novarum - sur la condition des ouvriers - que l'on considère comme «la grande charte» et «la somme de la doctrine sociale de l'Église» ${ }^{36}$. La onzième session, en 1932, étudie Quadragesimo Anno - sur la restauration de l'ordre social - et en 1938, la seizième traite de Divini Redemptoris - contre le communisme - Les sessions intercalées entre celles-ci en reprennent différents aspects et proposent des solutions aux problèmes sociaux à partir des encycliques. De même, l'École reproduit intégralement les différents textes pontificaux dans ses brochures; elle les commente, les explique et tente de les appliquer à la réalité québécoise.

A ces textes de base, il faut en ajouter deux autres qui servent particulièrement de point de référence sur les problèmes du monde ouvrier. Il s'agit tout d'abord de l'encyclique Singulari Quadam écrite en 1912 et adressée aux évêques allemands. Pie X y donne des directives précises sur l'opportunité ou non de fonder des syndicats confessionnels dans les milieux composés d'ouvriers catholiques et de non-catholiques. Elle servira d'argument d'autorité en d'innombrables occasions pour convaincre le travailleur québécois. Enfin, la lettre de la Sacré Congrégation du Concile à Mgr Liénart, évêque de Lille, publiée en 1929, reprend les mêmes thèmes et servira aux mêmes fins.

L'École Sociale Populaire se veut le lien entre l'Église de Rome et l'ouvrier catholique québécois. Au-delà des publications périodiques, les cercles d'étude, l'École de formation sociale et les retraites fermées unissent plus étroitement l'E.S.P. et les dirigeants syndicaux. Le témoignage d'Alfred Charpentier est révélateur à ce sujet:

35 J.-P. Archambault, «Déclaration d'ouverture», Semaines sociales du Canada (Montréal, E.S.P., 1936), 19.

${ }_{36}$ Idem, "L'Église et le Syndicalisme», Semaines sociales du Canada (Montréal, E.S.P., 1921), 4. Les textes de l'E.S.P. se rapportant au syndicalisme catholique s'y référeront et la citeront constamment. 
Le Père Archambault a été mon «vade mecum» pendant quarante ans. Depuis ma première retraite fermée en 1914 [...] je n'ai cessé d'être en étroit contact avec lui [...] Après avoir été mon directeur spirituel pendant plusieurs années, il fut mon entraîneur à l'apostolat ouvrier, mon guide et mon soutien $[\ldots]^{37}$.

\section{II - LA DOCTRINE SYNDICALE DE L'ÉCOLE}

Dans un discours prononcé lors de la Semaine sociale de 1921, l'abbé Philippe Desranleau - il sera évêque en 1936 - précise le rôle que l'École entend tenir face à l'organisation ouvrière:

Il [le syndicalisme] constitue une nouvelle étape dans l'évolution sociale. C'est peine perdue que de chercher à l'enrayer. Le mieux est de s'en emparer pour le régulariser, le moraliser et l'améliorer ${ }^{38}$.

Nous illustrerons plus en détail cet effort de l'École en reprenant les thèmes principaux qu'elle a développés dans ses périodiques. Nous les avons regroupés selon les différentes valeurs auxquelles ils faisaient appel, soit le politique, le religieux, l'ordre et la paix sociale, la promotion professionnelle de l'ouvrier et la lutte aux «idéologies subversives». Il s'agira d'expliquer le rôle de ces thèmes dans la promotion du syndicalisme catholique.

\section{L'évolution de la thématique 39}

Comme l'indique l'analyse thématique de sa propagande, les préoccupations de l'École évoluent avec les années.

Les arguments religieux et moraux occupent la place la plus importante dans ses publications jusqu'à la fin des années 30 . Ce sont certes ceux qu' on connaît le mieux et qui, en dernière analyse, sous-tendent toute la logique de la promotion du syndicalisme au Québec. Cependant, ce genre de plaidoyer connaît une importance

37 Alfred Charpentier, "L'apôtre des travailleurs», Lettres du Bas-Canada, 21, no 1 (mars 1967): 43.

38 Philippe Desranleau, «L'organisation professionnelle», Semaines sociales du Canada (Montréal, L'Action Paroissiale, 1921), 92. C'est nous qui soulignons.

39 Pour une explication détaillée de la méthodologie employée, voir JeanClaude St-Amant, L'École sociale populaire et le syndicalisme catholique 1911-1949, thèse de maîtrise, Université Laval, 1976, 6-12. 
toujours décroissante par rapport aux autres thèmes. Nous assistons à l'introduction graduelle d'une argumentation nouvelle pour se gagner la faveur des travailleurs québécois.

D'autre part, notre analyse fait ressortir l'emploi constant d'arguments touchant la promotion professionnelle de l'ouvrier. Ces thèmes occupent soit le deuxième, soit le troisième rang. Cette constance tient surtout aux nombreuses interventions des cadres ou des aumôniers syndicaux aux Semaines sociales; les syndicalistes actifs tiennent un langage beaucoup plus pragmatique que ne l'est celui des religieux rattachés à l'École. Fait à première vue singulier, les années de crise économique n'amènent pas une augmentation sensible dans ce genre de préoccupation. On se rend tout simplement compte que le syndicalisme est impuissant contre les effets de la crise.

Les arguments touchant l'ordre et la paix sociale gardent un caractère accessoire tout au long du demi-siècle. Ils ont cependant été employés plus souvent entre 1920 et 1929 ainsi qu'après 1940. Les activités ouvrières au Québec et dans l'Ouest canadien dans le premier cas, la multiplication des cessations de travail par les syndicats dans le deuxième cas, semblent expliquer cette évolution. Dans la première période, l'accent porte sur le fait que les syndicats catholiques ne provoquent pas de grèves; quant à l'agitation des années 40, l'E.S.P. prétend alors que des organisations ouvrières catholiques plus fortes pourraient changer cette situation.

Les thèmes politiques sont plutôt négligés avant 1937. Il faut attendre la publication du Programme de restauration sociale et la poussée corporatiste de l'École pour que ces thèmes prennent une certaine ampleur. Le rôle du syndicalisme dans l'organisation professionnelle est alors discuté avec insistance et présenté soigneusement au lecteur. Le journal L'Ordre nouveau et, à un moindre degré, la revue Relations se chargent de transmettre ce message.

La lutte aux «idéologies subversives» occupe l'E.S.P. à divers degrés au cours de son histoire. Avant 1929, les auteurs d'articles s'attachent beaucoup plus à combattre les syndicats neutres alors que ceux-ci sont taxés de "bolchévistes» ou de socialistes. Ensuite, le communisme et le socialisme deviennent les théories que l'on combat avec acharnement et les périodiques de l'École privilégient ce thème. Les rédacteurs se sont servis de la religion pour combattre les unions neutres, mais s'en sont pris directement au socialisme et au communisme après 1929, quand ceux-ci ont semblé donner si- 
gne de vie au Québec. L'École les percevait comme une menace immédiate plus contre toute la société que contre les seuls travailleurs québécois. Les nombreux tracts et brochures s'y rapportant directement l'attestent.

\section{L'Idéologie syndicale}

L'E.S.P., œuvre jésuite, sent maintes fois le besoin d'expliquer ses prises de position sur un terrain que plusieurs considèrent de nature purement économique. À ceux-là, elle rappelle que la question sociale est "avant tout une question morale et religieuse qui ne pourrait être résolue que d'après les règles de la morale et le jugement de la religion» et qu'on «ne saurait être chrétien dans sa vie privée et paiën dans les affaires ${ }^{40}$.

Fidèle à cette ligne de pensée, l'E.S.P. souligne que la neutralité en matière syndicale est un ennemi subtil et extrêmement dangereux. L'ouvrier membre d'un syndicat neutre «tombe dans l'indifférence religieuse [...], il ne se rend pas compte que la neutralité religieuse a matérialisé son esprit»; l'étape suivante vers l'antireligion est vite franchie: "l'union neutre [...] est ou sera, à brève échéance, une union anticatholique ${ }^{41}$.

L'E.S.P. voit une autre bonne raison pour favoriser les organismes catholiques. La nécessité d'une saine orientation des forces ouvrières, que seule l'Église peut dispenser, se traduira par le rappel des devoirs des ouvriers envers leurs patrons: "rendre à chacun son dû, accomplir diligemment sa tâche quotidienne, respecter la parole donnée ${ }^{42}$. Elle insiste pour dire que ce sont là des responsabilités sociales que n'acceptent pas les groupements ouvriers d'origine américaine. On comprendra que cette manière de voir avait pour effet de rassurer quelques patrons et de les convaincre d'accepter la syndicalisation de leurs employés par une organisation catholique.

L'organisation ouvrière devient aussi un outil pour répandre la foi catholique; son inspiration chrétienne servira d'exemple pour les non-catholiques que l'on accepte dans quelques syndicats comme

40 «Chrétiens séparés: un pas vers l'union», Relations, 2, no 12 (décembre 1942): 319.

41 Edmour Hébert, L'Église et l'organisation ouvrière, 1.

42 E.S.P., Le syndicalisme national catholique (Montréal, E.S.P., 1937), 10, coll. «E.S.P.», no 285 . 
membres-adjoints, parce qu'ils seront noyés dans les milieux catholiques et prêts à en accepter les directives. De plus, elle servira à fortifier la foi des catholiques: "le syndicalisme catholique national est chez nous la plus effective "courroie de transmission" de la doctrine sociale de l'Église dans les masses populaires. C'est son grand mérite ${ }^{43}$.» Il faut comprendre que l'École entend utiliser la pratique syndicale pour conserver l'ascendance de l'Église sur la société en évolution rapide.

S'il est primordial pour l'École d'offrir une doctrine, elle juge important aussi que l'organisme qu'elle encourage puisse assurer une amélioration matérielle de la condition des travailleurs. Tout en se défendant bien d'en faire une préoccupation première, elle assure que ses «syndicats ne sont pas, comme certains le pensent, des congrégations pieuses; ils savent parler, débattre leurs intérêts» ${ }^{44}$. Ainsi, et c'est là son thème économique majeur, une adhésion nombreuse à l'organisation syndicale catholique permettra de réclamer "le droit à un salaire raisonnable pour l'ouvrier», "le relèvement économique du prolétariat», ou encore «l'abolition de la dégradation des pauvres ${ }^{45}$.

Ces buts seront atteints par la garantie de conditions de travail acceptables (durée, hygiène, travail de nuit limité, travail adapté à l'âge et au sexe...), par la mise sur pied de services d'aide aux travailleurs (bureaux de placement, caisses d'entraide, fonds pour les accidentés, formation des membres...), et par l'obtention d'augmentations de salaires.

Dans ce dernier domaine, la position affichée n'est ni constante ni très ferme. À preuve, cette déclaration d'un porte-parole des syndicats catholiques de Montréal, invité par l'École à s'adresser à un groupe de travailleurs: "Nos pères avec trente sous par jour élevaient une famille de vingt-six enfants. De nos jours, avec un salaire de famine nous en élevons douze» ${ }^{46}$; 1 'accent est mis sur le carac-

43 Jean d'Auteuil Richard, "L'enseignement social dans la C.T.C.C.», Relations, 1, no 6 (juin 1941): 161.

44 Alexandre Dugré «Souhaits de bonnes relations», Relations, 8, no 1 (janvier 1948): 1 .

45 J.T. Mc Nicholas, Mentalité communiste (Montréal, E.S.P., 1938), 19, coll. «E.S.P.», no 298.

46 M. Hogue, Compte rendu d'un discours prononcé lors d'une manifestation ouvrière, Semaines sociales du Canada (Montréal, L'Action paroissiale, 1921), 351. 
tère national des syndicats. Quelques années plus tard, en accord avec la C.T.C.C., l'École demande l'instauration du salaire familial. "C'est le salaire suffisant pour répondre aux exigences légitimes d'une famille ouvrière normale» ${ }^{47}$. Elle précise qu'il serait payé indifféremment à l'homme marié ou au célibataire, ou encore à la femme si elle fait un travail d'homme. "La normalité d'une famille ouvrière » et l'étendue de ses «exigences légitimes » ne sont pas explicitées davantage.

Le dynamisme des syndicats est maintes fois répété et, en 1938, l'E.S.P. soutient que «leur influence et leurs actes depuis 25 ans [...] constituent non seulement une promesse, mais une solide garantie » ${ }^{48}$. En contrepartie, les propagandistes de l'École soulignent la faillite des syndicats internationaux.

L'E.S.P. affirme que ses efforts tendent à remédier au «régime antisocial de l'individualisme» autant chez les ouvriers que chez les patrons. Comme les syndicats catholiques doivent donner l'exemple, elle souligne en 1937 l'affiliation prochaine de ceux-ci à une organisation internationale des travailleurs chrétiens. La puissance qu'ils en retireront devrait suffire à gagner la confiance de la population ouvrière ${ }^{49}$. De même, la présence de syndicats catholiques à la Conférence Internationale du Travail, à Genève, la remplit d'aise... et alimente sa propagande ${ }^{50}$.

Tout en dénonçant les abus du capitalisme, les revendications de l'E.S.P. sont habituellement faites en spécifiant du même coup que le respect de la propriété privée est absolue chez les syndicats catholiques et que la répartition inégale des richesses est reconnue comme nécessaire. Inutile de dire que ces précisions atténuent la portée de leurs réclamations. Ce n'est que vers les années 40 que l'E.S.P. reconnaît aux syndicats, et timidement encore, un rôle vraiment revendicateur: «le syndicat se distingue [...] premièrement par sa fin propre et directe, qui, pour être subordonnée aux fins su-

47 «Plan d'étude», L'Ordre nouveau, 3, no 5, (5 décembre 1938).

48 Omer Genest, «Les syndicats catholiques et nationaux», L'Ordre nouveau, 3, no 4 (20 novembre 1938).

49 "L'internationale syndicale chrétienne», L'Ordre nouveau, 2, no 3 (5 novembre 1937).

so Pierre Beaulé, Compte rendu d'un discours prononcé lors d'une manifestation ouvrière, Semaines sociales du Canada (Montréal, E.S.P., 1931), 302. 
périeures de l'ordre religieux et moral, n'en est pas moins d'ordre économique ${ }^{51}$.

Le syndicalisme catholique doit présenter une image sécurisante si on pense le faire accepter de tous. Conformément à sa doctrine, l'E.S.P. le décrit comme respectueux de l'ordre et garant de la paix sociale.

En ce sens, l'École établit dans un premier temps le droit à l'existence des syndicats dans la société. Selon elle, ce droit leur a été octroyé par la nature même. Elle fait aussi appel à l'histoire pour justifier leur présence: la révolution française a injustement supprimé les corporations (ancêtres des syndicats) sans pour autant s'assurer que les ouvriers soient suffisamment protégés, il incombe à la société moderne de remédier à cette lacune.

Paradoxalement, l'E.S.P. pose ses conditions à la reconnaissance de ce «droit naturel» pour tous les syndicats. Ceux-ci doivent se donner une existence légale par l'incorporation, car elle est «une garantie d'ordre et de sécurité pour les ouvriers, pour l'industriel et pour la société elle-même» ${ }^{52}$. Sa position s'explique quand on sait que seuls les syndicats catholiques ont accepté l'incorporation. L'E.S.P. joue sur ce facteur pour les favoriser au détriment des syndicats internationaux qui, eux, sont en «coalition contre l'ordre public».

Insérée à sa juste place dans la société, l'organisation ouvrière catholique gardera la famille unie; le Petit catéchisme d'éducation syndicale affirme que "grâce au syndicat, les ouvriers peuvent obtenir [...] des salaires convenables, permettant à leur femme de rester à la maison pour élever en paix leurs enfants $" 53$.

Les syndicats catholiques aspirent aussi à «établir des relations cordiales et harmonieuses entre patrons et ouvriers, entre le capital et le travail ${ }^{54}$. Ils deviennent ainsi des «instruments de pacification sociale» car ils ont appris que «défense professionnelle ne dit pas

51 Alphonse Allard, Compte rendu d'un discours prononcé lors d'une manifestation ouvrière, Semaines sociales du Canada (Montréal, E.S.P., 1940), 169.

52 Richard Arès, Petit catéchisme d'éducation syndicale (Montréal, E.S.P., 1938), 25, coll. «E.S.P.», no 295.

53 Ibid., 9.

54 Henri Beauvais (pseudonyme de J.-P. Archambault), L'Église et les associations ouvrières (Montréal, E.S.P., 1913), 14, coll. "E.S.P.», no 17. 
nécessairement lutte des classes ${ }^{55}$. Dans cette même ligne de pensée, l'E.S.P. prétend que le syndicat catholique évitera les grèves. Celui-ci entend épuiser d'abord tous les autres moyens de conciliation ne recourant que rarement et dans le cas de nécessité seulement à la cessation de travail. Si le droit de grève est considéré comme légitime, l'École précise qu'il n'est pas nécessairement lié au droit syndical, ce qui se traduit, par exemple, par sa désapprobation de la grève des policiers et celle des employés des transports en commun à Montréal en 1943 et 1944. Sur cette question, l'approbation ou non d'une grève par l'E.S.P. dépend presqu'exclusivement du statut confessionnel du syndicat qui la fait.

L'E.S.P. prétend également que l'ouvrier membre de l'organisation qu'elle défend "peut même protéger les patrons honnêtes contre la concurrence ruineuse de quelques-uns de leurs collègues » ${ }^{56}$. Cependant, l'attitude de l'École vis-à-vis des patrons évolue avec les années. En 1932, elle se plaint que:

un effort considérable a été fait depuis quinze ou vingt ans par le syndicalisme catholique et national [en vue d'intéresser les patrons au mouvement]. Malheureusement, l'effort a été unilatéral. Nos patrons n'ont à peu près pas bougé, pas même les catholiques $^{57}$.

Six ans plus tard, sa position est devenue nettement plus dure. Face aux employeurs qui invoquent la liberté de travail pour refuser toute syndicalisation, un rédacteur de L'Ordre nouveau écrit:

le développement du syndicalisme est dû, pour une part, aux excès, aux abus du monde patronal. Les ouvriers ont compris [...] que la fameuse liberté de travail dont on veut les gratifier n'est en somme que la liberté de crever de faim, ou d'être exploités honteusement ${ }^{58}$.

Ce n'est pas sans une pointe d'amertume qu'elle avoue finalement la faillite du syndicalisme sur ce plan:

Le syndicat, si nécessaire qu'il puisse être, est surtout une arme de combat, ce n'est pas en soi un outil de reconstruction so-

55 J.-P. Archambault, Le syndicalisme catholique au Canada, 3.

s6 J.-P. Archambault, l'Église et l'organisation ouvrière (Montréal, Oeuvre des tracts, 1919), 10, coll. "Oeuvre des tracts», no 8.

57 Émile Cloutier, «L'organisation professionnelle», Semaines sociales du Canada, (Montréal, E.S.P., 1932), 265.

58 "Conseils du travail», L'Ordre nouveau, 2, no 14, (20 avril 1938). 
ciale [...] De toute façon, la formule syndicale s'avère impuissante à assurer de façon stable la paix entre patrons et employés ${ }^{59}$.

La volte-face de l'École sur cette question, bien que lente, semble se faire sous l'influence des syndicats catholiques. De fait, l'organisation syndicale, devenue nettement plus militante dans les années 40, pousse l'École à durcir ses prises de position.

Toujours par souci d'ordre et de paix, l'E.S.P. accepterait théoriquement la collaboration entre divers organismes ouvriers.

[Quant aux] cartels intersyndicaux entre syndicats chrétiens et syndicats neutres ou même socialistes, qu'on se rappelle que ce n'est permis qu'à titre exceptionnel et temporaire ${ }^{60}$.

À ce sujet, la position de l'E.S.P. reste empreinte d'une méfiance manifeste.

L'E.S.P. reproche aux chefs syndicaux américains de diriger les destinées des ouvriers à partir de New York ou de Chicago et de s'enrichir avec les cotisations syndicales du pays, sans pour autant aider la cause des travailleurs. Un syndicalisme indépendant au Québec constituerait un exemple à suivre dans les autres provinces. Ce genre d'argument prend facilement la forme de nationalisme. Ainsi, le syndicat catholique servira efficacement de moyen de défense de la langue et des traditions, parce qu'il est composé en majorité de Canadiens français. Sont inclus alors parmi les adversaires «les révolutionnaires de l'ouest» à qui on reproche leur adhésion à la «One Big Union» en 1919, ou encore les Ontariens, anglais et protestants «qui se noient à plaisir dans l'américanisme» ${ }^{61}$. Dans la même veine, on reproche aux syndicats internationaux de mettre la force de leur organisation au service de la centralisation.

Ces thèmes ne disparaîtront jamais complètement, mais ils seront relégués à l'arrière-plan dans les années 30. Une nouvelle

59 Jean-Baptiste Desrosiers, Compte rendu d'un discours prononcé à une assemblée ouvrière, Semaines sociales du Canada (Montréal, E.S.P., 1943), 171. La même année est fondée l'Association Professionnelle des Industriels en collaboration avec le père Bouvier. On avait tout d'abord souhaité réunir patrons et ouvriers dans une même organisation.

60 E.S.P., La charte officielle du syndicalisme chrétien (Montréal, Oeuvre des tracts, [1929], 2, coll. "Oeuvre des tracts», no 123. Cette déclaration s'inspire directement de la lettre de la Sacrée Congrégation du Concile à $\mathbf{M}^{\mathrm{gr}}$ Liénart, évêque de Lille, parue en 1929.

${ }^{61}$ Alfred Charpentier, L'aube d'une ère ouvrière nouvelle (Montréal, E.S.P., 1922), 13, coll. «E.S.P.», no 104. 
argumentation les remplacera pour gagner la faveur de la population ouvrière de la province.

Tout d'abord, la méfiance de l'École envers l'État, clairement exprimée à plusieurs reprises, encourage celle-ci à présenter les syndicats comme des groupes de pression appelés à influencer la législation en faveur des travailleurs. "La plupart des lois sociales votées depuis quelques années par la législature du Québec ont là leur origine ${ }^{62}$, assure le père Archambault en avril 1936. L. Girard, syndicaliste catholique de Montréal, réclame d'ailleurs pour son organisme la paternité de la loi provinciale de l'extension juridique de la convention collective de travail, votée en $1934^{63}$. Cette dernière est très appréciée et l'E.S.P. l'utilise souvent pour faire sa publicité.

L'E.S.P. insiste cependant sur l'indépendance des syndicats catholiques envers les partis politiques. Elle précise que:

dès qu'un syndicat se rattache à un parti politique, il perd son caractère de syndicat pour devenir un groupe d'ouvriers qui travaillent au triomphe d'un parti dont ils espèrent tirer profit pour leurs intérêts ${ }^{64}$.

Avec plus de fermeté encore, l'École se défend bien de laisser le sort des travailleurs aux mains des politiciens et les rédacteurs de L'Ordre nouveau de s'exclamer: "Quand donc règlerons-nous nos problèmes sociaux en dehors de toute politique $\|^{65}$. L'École voudrait éviter la partisanerie politique et «remplacer l'État dans les tâches pour lesquelles il n'est point fait ${ }^{66}$.

Le besoin de l'E.S.P. de se désolidariser des partis politiques se fait particulièrement sentir après la prise de pouvoir de Duplessis.

62 J.-P. Archambault, Le syndicalisme catholique au Canada (Montréal, E.S.P., 1936), 19, coll. «E.S.P.», no 267.

63 E.S.P., Journées anticommunistes, II: 12. Cette loi étend à toutes les industries semblables d'une même région une convention collective signée par un syndicat, que les employés soient syndiqués ou non. Voir Céline Saint-Pierre, "Idéologies et pratiques syndicales au Québec dans les années 30: La loi de l'extension juridique de la convention collective de travail», Sociologie et sociétés, 7, no 2 (nov. 1975): 5-32. 273.

64 «Horizon international. Costa Rica», Relations, 3, no 10 (octobre 1943):

65 "Liberté de travail vs atelier fermé», L'Ordre nouveau, 2, nos 19 et 20 (5 et 20 juillet 1938).

66 Émile Cloutier, "L'organisation professionnelle», Semaines sociales du Canada (Montréal, E.S.P., 1932), 255. 
Ceci s'explique pour qui connaît l'épisode du Programme de restauration sociale, publié par l'École et adapté par l'Action Libérale Nationale. La rupture entre cette dernière et Maurice Duplessis, après l'élection de 1936, incite l'E.S.P. à répéter sa neutralité face aux partis politiques.

Un dernier thème important découle de la doctrine corporatiste mise de l'avant par l'E.S.P. L'École se fait un devoir de rappeler que les syndicats de travailleurs constituent une composante essentielle dans l'organisation professionnelle. Celle-ci «comprend tous ceux qui coopèrent à l'exercice d'une même profession, c'est-à-dire les patrons et les ouvriers des diverses sections constituant, selon la nature de leur travail une même profession " ${ }^{67}$. Une fois toutes les professions organisées sur une telle base et les liens assurés entre elles, le régime corporatiste sera institué et le rôle de l'État se bornera à «favoriser la libre action des divers éléments et [à] n'intervenir que pour suppléer à leurs déficiences ou équilibrer leur activité» ${ }^{68}$.

Les "éléments mauvais et étrangers", les "groupements dangereux et les fausses théories», les «idées subversives» que combat l'École à l'aide du syndicalisme catholique peuvent se résumer aux deux termes utilisés le plus fréquemment pour les désigner: socialisme et communisme. L'E.S.P. inclut aussi le «collectivisme» dans la définition qu'elle en donne en 1932:

[...] trois mots qui s'emploient souvent l'un pour l'autre. Ils désignent un ensemble de systèmes économiques qui attribuent à la communauté ou société, à l'exclusion des particuliers, la propriété des biens matériels, principalement des biens de production ${ }^{69}$.

À partir de cette formulation, on situe le socialisme à droite du mouvement et le communisme à gauche.

Sur le plan pratique, l'identification précise des adversaires demeure plus confuse. On se souvient avoir soutenu «une bataille des idées» avec «certains radicaux et trois cents Russes» lors de la fondation de l'union ouvrière de Thetford en 1913. Plus d'un in-

67 "Le régime purement syndical est impossible», L'Ordre nouveau, 3, nos 19 et 20 ( 5 et 20 juillet 1939 ).

68 Omer Genest, «Le syndicalisme a-t-il vécu?», L'Ordre nouveau, 3, no 4 (20 novembre 1938).

69 E.S.P., Cahier anticommuniste (Montréal, E.S.P., 1932), 3, coll. «E.S.P.», nos 217-218. 
dice peut servir à démasquer les coupables; le simple fait de ne pas critiquer les communistes ou les socialistes semble être suffisant pour être considéré sympathisant (sinon complice) à leur cause. Sur des preuves tout aussi tangibles, l'École s'en prend à tous les syndicats non-catholiques:

Qu'ils s'appellent Syndicats nationaux, Syndicats internationaux, la Fédération canadienne du Travail, la Fédération américaine du Travail, ou la One Big Union, [ils] sont tous d'une façon plus ou moins consciente, minés par le socialisme ${ }^{70}$.

L'École se méfie énormément de la tactique d'infiltration que les «rouges» emploient pour prendre la direction des syndicats (elle en connaît l'efficacité pour l'avoir employée elle-même à son avantage). Ainsi, la décision de la centrale communiste "Workers Unity League» de se dissoudre en 1935 est purement stratégique; elle survient après que ses membres eussent été suffisamment endoctrinés pour répandre leur message dans toutes les autres organisations de travailleurs. Il est facile de conclure ensuite que «si la C.T.C.C. n'était pas une organisation anticommuniste, elle perdrait sa raison d'être ${ }^{71}$.

Il est évident que dans cet esprit l'E.S.P. applaudit à la «loi du cadenas» votée par le gouvernement Duplessis en 1937 et dirigée (en théorie) contre les communistes:

Celle-ci $[\ldots]$ a été appliquée en diverses circonstances avec d'heureux résultats $[. .$.$] Les catholiques ont, eux, accueilli com-$ me sage et salutaire cet acte législatif qui a reçu la pleine approbation de l'épiscopat ${ }^{72}$.

L'E.S.P. n'use d'aucun ménagement quand elle peut identifier nommément quelque communiste. Ainsi, les dirigeants syndicaux Kent Rowley et Madeleine Parent, accusés en 1946 de conspiration et de sédition, sont invités «à s'en aller à Moscou, où ils finiront par devenir d'authentiques travailleurs dans quelque camp de concentration»; la leçon qu'il faut en tirer suit immédiatement: «donnons notre confiance aux organisations de chez nous» ${ }^{73}$.

70 Philippe Desranleau, «L'organisation professionnelle», Semaines sociales du Canada (Montréal, L'Action paroissiale, 1921), 84.

71 "Le congrès de la C.T.C.C.», Relations, 9, no 10 (oct. 1949): 270.

72 «Liberté de travail vs atelier fermé», L'Ordre nouveau, 2, nos 19 et 20 (5 et 20 juillet 1938 ).

73 «Valleyfield», Relations, 6, no 9 (sept. 1946): 282. 
L'indignation de l'École est à son comble devant l'élection à Montréal de Fred Rose comme député fédéral du Parti ouvrier progressiste (communiste) en 1943. L'École suit d'ailleurs de très près les élections, tant fédérales que provinciales. Les votes communistes constituent la preuve irrécusable que, «depuis trente ans, les bolchéviques ont mis sur pied des organes de propagande et d'agitation internationales dont l'efficacité n'est plus à démontrer ${ }^{74}$.

\section{CONCLUSION}

L'E.S.P. dispose d'une doctrine bien définie et elle entend l'utiliser pour démontrer la justesse de ses objectifs dans le monde ouvrier. Le syndicalisme catholique que l'on propose est devenu à maints égards du catholicisme syndical où la doctrine catholique a primé sur la pratique syndicale.

Si cette affirmation reflète la réalité des Jésuites et du groupe laïque qui gravite autour d'eux, l'ensemble du clergé n'a pas toujours vu le besoin de s'impliquer aussi activement. Encore en 1918, le père Archambault a voulu secouer l'inertie du monde clérical en rédigeant un volume consacré à l'action sociale ${ }^{75}$. Il s'adresse directement aux prêtres et tente de les convaincre de la nécessité pour eux de s'engager dans des auvres à caractère social. Le syndicalisme catholique y occupe une place importante et l'effort concentré vers une élite laïque constitue le moyen suggéré pour une implication sociale efficace.

Cette dernière optique sous-tend constamment les efforts de propagande de l'École. Un élitisme certain est présent lors de la création de cercles d'études qui se donnent pour but de former un groupe d'ouvriers prêts à fonder des syndicats catholiques. Il se manifeste aussi à la fondation des Semaines sociales du Canada. L'E.S.P. entend y "former une élite qui fera revivre l'esprit chrétien dans les mœurs, les institutions et les lois » ${ }^{76}$. L'École de formation sociale est destinée à préparer des chefs catholiques dans les milieux ouvriers et l'Ordre nouveau avoue aussi aller dans la même direction.

\footnotetext{
74 «Fédération mondiale des syndicats», Relations, 9, no 9 (sept. 1949) : 250.

75 Joseph-Papin Archambault, Le clergé et l'Action sociale (Montréal, E.S.P., 1918).

76 «Les Semaines sociales», L'Ordre nouveau, 4, no 23 (5 sept 1940).
} 
Le message de l'École que nous avons analysé a donc certainement eu son plus grand impact au niveau de l'élite.

L'École rencontre «l'ennemi» au début des années 30: le communisme. Une exposition positive de la doctrine catholique ne semble plus suffire. Les propagandistes doivent s'acharner à dénoncer, à combattre et à détruire; les thèmes anticommunistes deviennent prédominants. Parallèlement à ce changement, l'École se dote de nouveaux moyens de propagande qui étendent son rayon d'action. Le service de presse, utilisé partout au Canada français, s'occupe principalement de lutte contre le communiste. L'École de formation sociale forme des spécialistes en anticommunisme qui répéteront son message à qui veut l'entendre. Une série de brochures et de tracts seront diffusés dans la population par l'entremise de «journées anticommunistes ", de causeries radiodiffusées, de sermons dans les églises et d'articles de journaux et de revues. Le journal de l'École qui paraît d'ailleurs durant ces années consacre une large part de ses énergies aux mêmes thèmes. L'anticommunisme constitue sans contredit le message que les Jésuites ont contribué à répandre avec le plus d'archarnement et d'efficacité. 